\title{
Persistent nepotism in peer-review
}

\author{
UlF SANDSTRÖM, ${ }^{\text {a }}$ MARTIN HÄLLSTEN ${ }^{b}$ \\ ${ }^{a}$ Department for Studies of Social Change and Culture, Linköping University,Lingköping (Sweden) \\ b Departmen of Sociology \& Swedish Institute for Social Research, Stockholm University, \\ Stockholm (Sweden)
}

\begin{abstract}
In a replication of the high-profile contribution by Wennerås and Wold on grant peer-review, we investigate new applications processed by the medical research council in Sweden. Introducing a normalisation method for ranking applications that takes into account the differences between committees, we also use a normalisation of bibliometric measures by field. Finally, we perform a regression analysis with interaction effects. Our results indicate that female principal investigators (PIs) receive a bonus of $10 \%$ on scores, in relation to their male colleagues. However, male and female PIs having a reviewer affiliation collect an even higher bonus, approximately $15 \%$. Nepotism seems to be a persistent problem in the Swedish grant peer review system.
\end{abstract}

\section{Introduction}

Gender bias in the grant peer-review procedures of research councils is a crucial issue that generates serious discussion in core scientific journals [WESSELEY, 1998; BORNMANN \& DANIEL, 2005]. Any type of bias would be detrimental towards strategies for scientific excellence. The proposed European Research Council is but one illustration of the importance of this debate. Since WENNERÅs \& WOLD [1997]

Received February 12, 2007

Address for correspondence:

ULF SANDSTRÖM

Department for Studies of Social Change and Culture, Linköping University

Lingköping, Sweden

E-mail: ulfsa@tema.liu.se

$0138-9130 /$ US $\$ 20.00$

Copyright (C) 2007 Akadémiai Kiadó, Budapest

All rights reserved 
published the first ever analysis of peer-review scores for postdoctoral fellowship applications no studies have been able to use the same variables, including reviewer affiliation.

This study aims to trace the influence of gender and conflict-of-interest on scores, controlling for performance measures (bibliometrics), academic status (professor, assistant professor, and researcher), experience (years since dissertation), faculty discipline (medicine or not), university affiliation and committee assignment.

\section{Research councils and peer review}

An essential principle of the classical model for research councils is that scientists, unrestricted by and unaffiliated with external factors, should govern research. In Sweden, active researchers constitute a majority on the council's board, i.e. in all bodies where applications are assessed and evaluated and grants decided upon. Committee members are chosen through an electoral process involving all members of the research community in the university system. Peer review is used to guarantee the quality and diversity of basic research. A specific feature of the Swedish councils is that scientific committees are in command of the work. As a consequence, and in comparison to the situation at NSF and NIH (main U.S. financing bodies), programme officers in Sweden have little or no influence over granting procedures, initiatives and priorities (see [SANDSTRÖM \& AL., 1997]).

Peer review is a documented, critical review performed by peers (persons having technical expertise in the subject matter to be reviewed) who are independent of the work to be reviewed. It is a tool that funding agencies (and programme managers) can use to obtain high-quality technical and scientific input for decisions on allocating resources. Peer review is intended to improve both the technical quality of projects in $\mathrm{R} \& \mathrm{D}$ and the credibility of the decision-making process. The independence of peers should make them more effective than internal reviewers.

Generally, the procedures among the councils in Sweden were similar during the 1990s. Each applicant (principal investigator) submitted a CV, bibliography and a research proposal. Applications were reviewed by one of 10 to 12 committees, each covering a specified research field or discipline. Each application was rated by several reviewers in the committee to which it was assigned. Reviewers graded the proposals and "track records" with a score, and that score formed the basis for the ranking used in funding decisions. Accordingly, review committees set up a conflict-of-interest protocol stating which members of the committee that have an affiliation with applicants. Affiliation might be maternal, kinship or supervisor relations. These protocols are open for public access according to Swedish legislation. 


\section{The gender gap}

During the first half of 1990s, success rates for applicants to the Swedish Medical Research Council (MFR) were marked by a significant gender gap. Women often had $10 \%$ lower rates than men did. A study published in 1995 by Wennerås and Wold (later a full study was published in Nature [WENNERÅs \& WOLD, 1997]) disclosed a gender bias in the evaluation of merits for postdoctoral fellowships at the MFR. Thereafter, a shift in policy was implemented. Practices among most other research councils changed, and the gap narrowed significantly during the second half of the 1990s (Figure 1).

Wennerås and Wold showed that female productivity was credited lower than male productivity in the evaluation of applications for postdoctoral fellowships $(\mathrm{N}=114)$ submitted to the Swedish MFR. The conclusion was clear: "Our study", they said, "strongly suggests that peer reviewers cannot judge scientific merit independent of gender." (1997, p. 341). Affiliation with a committee member further increased the chances of being funded. The disclosure of discriminating procedures and nepotism resulted in an intensive debate.

A detailed examination of male and female success rates within the research councils from 1989 to 2000 reveal that success rates for males were generally 5 to 10 percentage points higher than the success rates for females. This difference decreased after the 1995 turning point (Figure 1), which indicates a positive effect on female funding chances.

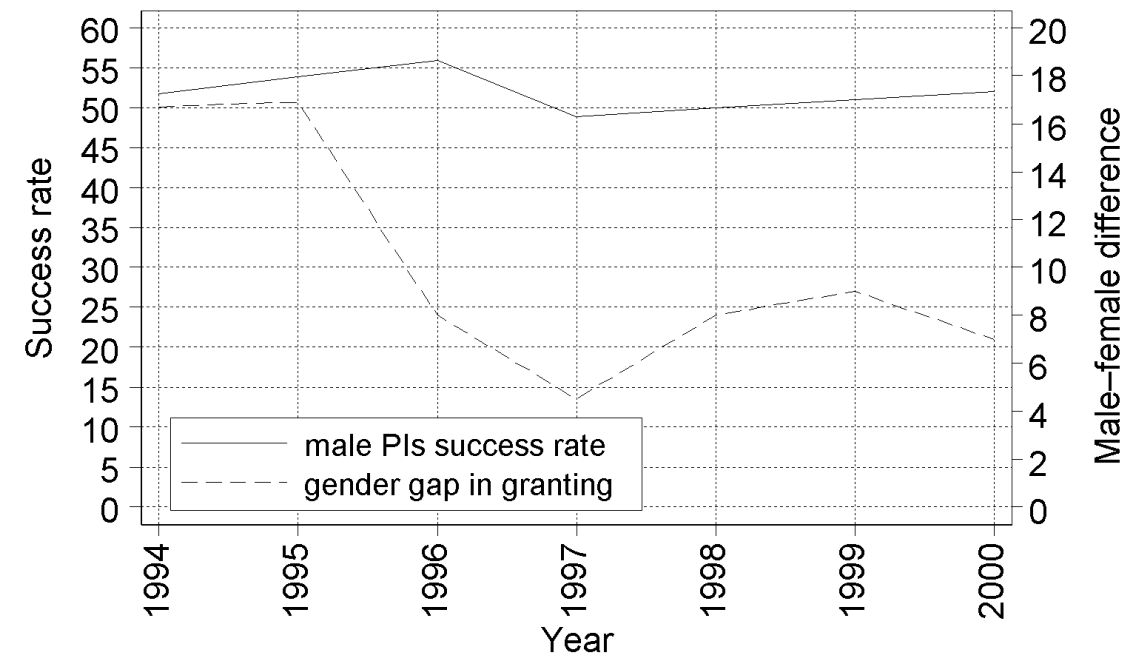

Figure 1. The Wold effect. Success rates of male applicants and the gender gap in the Medical Research Council 1994-2000 
This change in policy from the MFR could be called "the Wold Effect" However, the success rates for male applicants remained higher even after 1995. Obviously, the differences are partially explained by male domination in upper academic positions. Hence, a positive difference in success rates between males and females is not by itself an indication of discriminating procedures.

According to WOLD \& CHRAPKOWSKA [2004] policy changes already in 1997 were effective: "[I]ncreased knowledge on how prejudice influence peer decisions led to a reform of council procedure in 1997 so that it is now completely gender neutral" (our translation). We consider this as a controversial statement. A closer look at success rates for women indicate that structural factors for the group as distribution over committees and age/time elapsed since $\mathrm{PhD}$ etc. favour higher success rates, which leads toward closing the gender gap. Nevertheless, we will need a more comprehensive approach in order to fully understand the gender gap phenomena - our intention is to point at the nepotism factor which have not been investigated after the Wennerås \& Wold article. Lack of data is a considerable problem for researching that question, but as already indicated we have reliable data produced by the scientific committees themselves.

\section{Research on gender and Research Councils}

Gender in science is becoming a rather well-researched question [WESSELY, 1998]. Productivity issues was one of the first to be discussed (the productivity puzzle) by Cole \& ZuCKerman [1987], Long [1992], KyviK \& Teigen [1996] and XIE \& SHAUMAN [1998]. Further investigations have both challenged this discussion but also placed in wider context. There seem to be no significant differences when age, field and other variables are held constant; LEVIN \& STEPHAN [1998], PRPIC [2002], ASMAR [1999], BLACK \& HOLDEN [1998]. GANDER [1999] finds a higher marginal productivity among female researchers at liberal arts institutions. Results from a large sample of faculty from 57 universities in Netherlands indicated that factors affecting research productivity were identical for men and women, and that a family-related variable such as small children in the family, had no effects on research productivity. The territorial segregation issue, the gendered stratification of the scientific work force along disciplinary lines, has also raised interest. Rossiter [1978] and KULIS \& AL. [2002] are contributions to that literature. Women are overrepresented in fields like chemistry and microbiology, areas with high journal impact factors.

The paper by WENNERÅ \& WOLD [1997] is a landmark in research on gender bias. Their article is one of the most frequently cited in this area of research (http://garfield.library.upenn.edu/histcomp/peer-review_to/list/au-lcs.html). It should be underlined that they showed that bias due to nepotism was as strong as the gender bias effect. Unfortunately, virtually none of the papers citing their article have attempted to replicate nepotism issues. 
There are, however, a few exceptions to this observation: reports from funding agencies in the Netherlands and the UK have discussed fairness and funding in gender research from the perspective of modernising peer review. Other research projects have presented results from Denmark and the Netherlands [BROUNS, 2000]. However, none of these reports have been able to challenge the methodological excellence of the Wennerås \& Wold paper. Especially, their combination of data extracted from applications (council database), bibliometric data (from $\mathrm{CV}$ and publication list) and conflict-of-interest (protocol from committees) are unusual, and a replication after ten years is warranted.

In a thorough analysis MOED [2005, CH. 20] shows that in a national research council (the council was held anonymous) proximity between applicant and expert committee was a strong determinant for priority rating of the application. Being member of the committee was considered as the highest level of proximity. Using a large data set, output and field normalised impact figures for 3,300 applicants and committee members were calculated. Proximity was indexed according to whether the applicant or co-applicants were members of the committee. The study is relevant in this context, although the Swedish case is somewhat different, as data on conflict of interest is available.

Confidence in the peer review system is essential to the academic community. The legitimacy of funding procedures is based largely on trust. Therefore, conclusions about the functions and performance of research councils should be scrutinised and well researched. The problem with several of the studies on gender bias in research councils is that they cover only small samples and short periods of time i.e. mostly crosssectional studies. Although most of the work to date is careful and methodologically sound science, the lack of data is a general concern.

For the development of this area of research richness and quality of data is necessary. Generally, investigations on this subject have had problems in finding relevant data on the applicants and their research areas. Due to restrictions and economy of the research process, many of the studies have used only simple descriptors, e.g. age, time lapsed since dissertation, affiliation, publications, review grade, etc. as explanatory factors in relation to decisions taken by the councils. Now, we can add reliable data on reviewer affiliation, and advanced bibliometric analysis.

Table 1. Linear combinations of gender and conflict of interest coefficients

\begin{tabular}{|c|c|c|c|c|c|}
\hline & & \multicolumn{2}{|c|}{ male PI } & \multicolumn{2}{|c|}{ female PI } \\
\hline \multirow[t]{2}{*}{ no association } & point estimate & \multicolumn{2}{|c|}{0.000} & \multicolumn{2}{|c|}{0.097} \\
\hline & confidence interval & {$[-$} & $-]$ & {$[0.027$} & $0.167]$ \\
\hline \multirow[t]{2}{*}{ conflict of interest } & point estimate & \multicolumn{2}{|c|}{0.151} & \multicolumn{2}{|c|}{0.167} \\
\hline & confidence interval & {$[0.032$} & $0.271]$ & {$[0.051$} & $0.282]$ \\
\hline
\end{tabular}

Source: Table 2, model 10. 


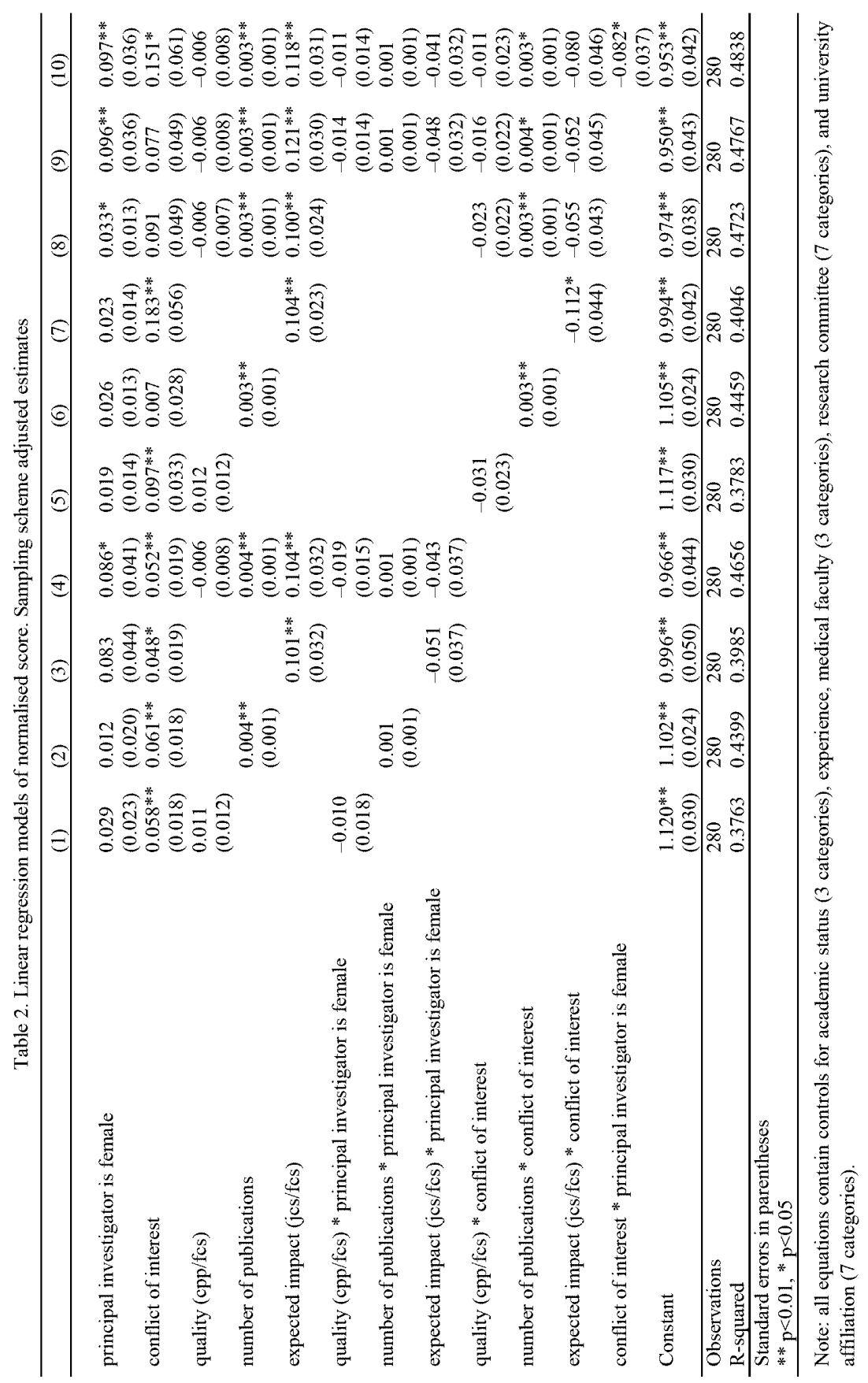


The 1990s were a dynamic period as regards Swedish research policy. New aspects to consider were introduced, e.g. gender, support of young researchers, interdisciplinary areas, strategic research etc. It was a period when research councils had to learn how to adjust to a changing environment. What has happened after ten years of time? Are female investigators still discriminated? Is reviewer affiliation no longer, as Wold has suggested, a disturbing factor in peer decisions on grant applications?

\section{Data and methods}

Our research draws on data from the sub-council for Medicine (a division of the Swedish Research Council) on the research grant applications submitted, reviewed and acted upon during 2004. A stratified sample was drawn from the 611 proposals for new projects, $63 \%$ of which had been submitted by male applicants. The Scientific Council for Medicine consists of eleven committees representing different medical disciplines or areas of research. Since female applicants were under-represented, and our primary interest is the effect of gender, we over-sampled women and adjusted our estimates accordingly. Our data include 280 applications, of which 118 are from female principal investigators (PIs). Beside the new projects a number renewals are reviewed each year. Renewals are projects that had funding the earlier two or three year period (see Figure 2).

Applicants submit an electronic research proposal to the Council. Each proposal is scored by 5 of the 6 reviewers on the committee to which the application is assigned. Reviewers assign a score between 1 and 7 on the following parameters: scientific competence, methodology and research question. Then an average for each parameter yields a final score on which the ranking is based. Policies of secrecy within the Scientific Council for Medicine classify "white sheets" from committee meetings as non-public documents. Consequently, we do not have the scores on scientific competence, the parameter used by Wennerås and Wold. Instead, we use the final ratings that are good estimates of competence scores (for 313 applications in year 2005, $r=0.90$ ).

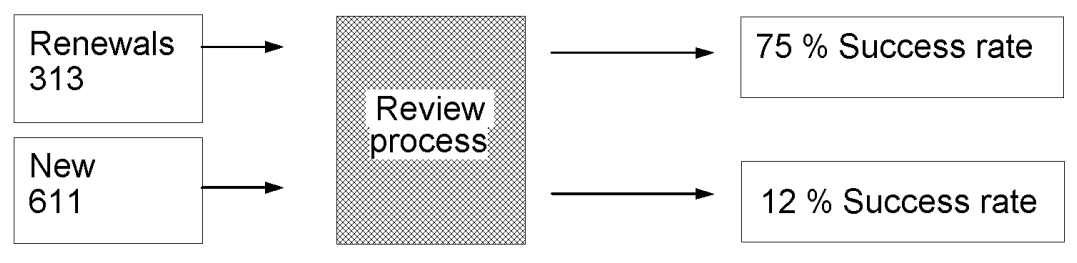

Figure 2. The review process of the Swedish Medical Research Sub-Council in 2004 
Rating practises differ among committees. Our methodology to compensate for this is to normalise all scores within each committee in relation to the committee mean value. Wennerås and Wold were unable to normalise because they would have needed data on all projects to produce a committee average. However, as they had only data on applications for post-doctoral fellowships they could not create a normalised score per committee.

Figure 3 describes the distribution of grades for applications in the council's committees. It is evident that scoring practises differ across committees with respect to mean value, variance and overall shape of the distribution. A normalisation procedure is thus necessary to adequately compare applications form different committees. We normalise by dividing each score by its committee's mean value, hence the normalised grade range from 0.6-1.6. Note that the mean value is calculated for all applications in the committee, not only our sample. We will interpret the grade points as percentage points. The dependent variable (normalised grade) ranges between 0.66 and 1.67 (with mean 1.16 and standard deviation 0.178 ). Since the range is very close to 1 , the coefficients*100 can be interpreted as percentage point changes.

The Swedish Research Council's conflict-of-interest policy specify that committees shall not review applications submitted by their own members, and that any such applications shall be re-assigned to other committees. Reviewers who declare a conflict of interest, normally due to supervisor, collaborative or kinship associations, are not permitted to score the proposal in question. Conflict-of-interest data are available by virtue of the principle of public access to official records.

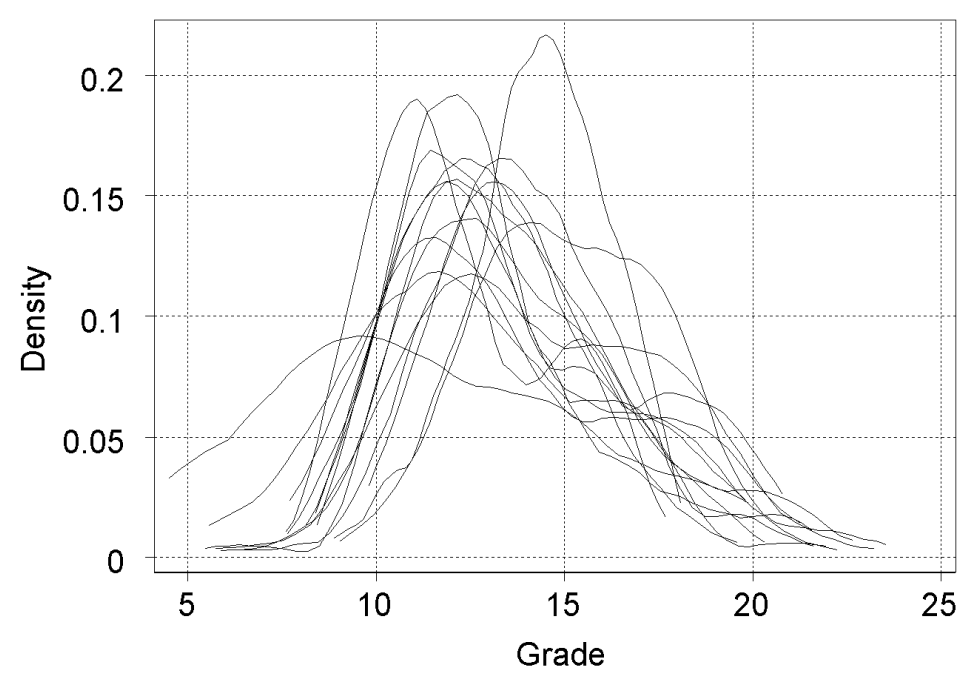

Figure 3. The distribution of grades in different committees prior to normalisation. Kernel density estimates 
Full bibliometric information for publications from 1998 through 2004 was collected from the publication lists attached to the proposals. We double-checked against Internet Web of Science to have a secure basis for the bibliometric measures. To determine whether a certain volume of citations is high or low, it is necessary to measure against an international reference value. Journal citation score (JCS) is the average citation rate of all papers in journals where a researcher has published. Field citation score (FCS) is an average citation rate based on all journals within a specific subject code. These codes were produced by Thomson/ISI in order to classify journals. The JCS/FCS ratio gives the expected relative citation impact for journals, which is a more stable indicator of journal quality than the impact factor from Thomson/ISI. As an additional performance measure, we use number of papers $(\mathrm{P})$.

The measured impact of a researcher, the number of citations per publication (CPP), concerns the actual citation rate. We apply standard bibliometric methods with fieldnormalised impact, e.g. CPP/FCS gives a relative figure of citation rates [VAN RAAN, 2006] Reference values, designed for each type of article (article, letter note or review) and for each year, is the basis for all subsequent ratios. For the analysis we use a twothree year citation window. We have not been able to correct for self-citations [GLÄNZEL \& AL., 2006].

Having data on all journals in each category, we can determine whether citation performance is higher or lower than the field average. With the FCS indicator, we also have a more precise description of scientific field than Wennerås and Wold. They classified researchers in ten fields according to their main area of research. Web of Science classification of journals in which articles are published provides much more detailed information, and we can benefit from this field indicator.

\section{Results: Nepotism in the medical sub-council}

Are there systematic differences in ratings between men and women, and if so, which are the determining factors? First of all, we examine how grading procedures is related to gender of the applicant. To fully asses the impact of nepotism and gender on grading procedures, it is important to explore all interactions with productivity. Figures $4 \mathrm{a}-\mathrm{c}$ show the relation between quintiles of productivity measures and awarded scores.

For citations per paper (Figure 4a), the awarded score increases with productivity for male PIs but not for female PIs. Instead, female PIs are rewarded the same score regardless of productivity.

In Figure 4b, male PIs seem to be reward higher grades than female PIs in the highest quintile of the impact score, but female PIs seem to have small advantage in the lower quintiles. In Figure 4c, awarded scores increase with number of publications to a similar extent for male and female PIs. 
U. SANDSTRÖM, M. HÄLLSTEN: Persistent nepotism in peer-review

a)

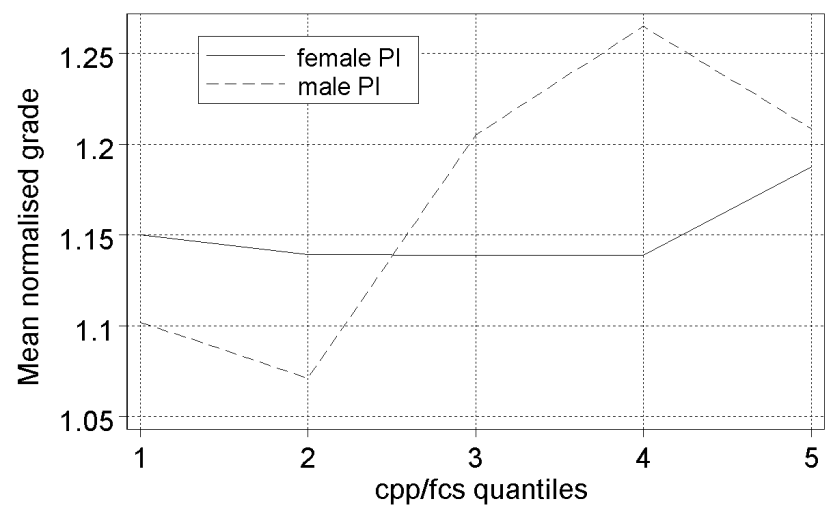

b)

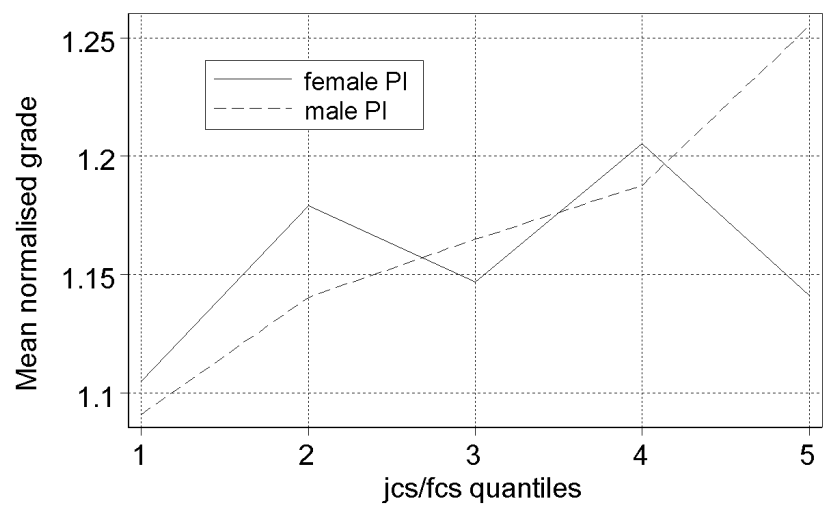

c)

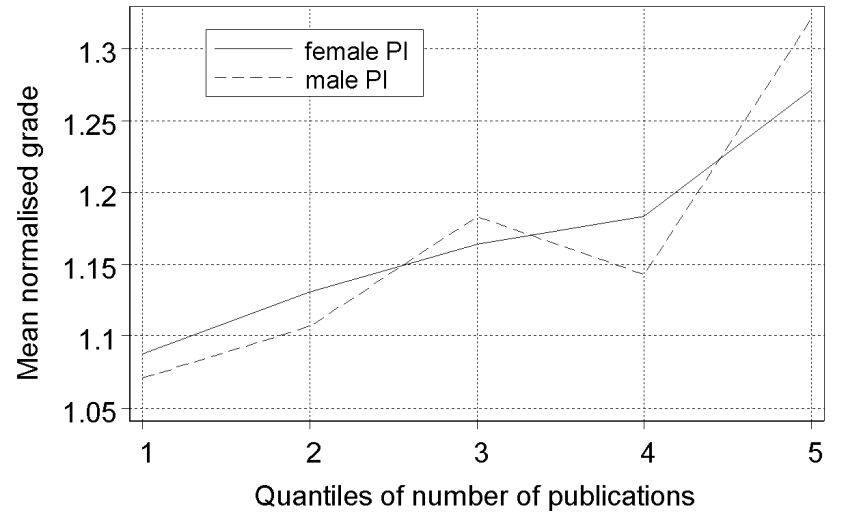

Figure 4. The association between grade and three different productivity measures in quantiles. a) Citations per paper (CPP/JCS); b) Journal Impact (JCS/FCS); c) Number of publications Separate estimates for male and female principal investigators 
U. SANDSTRÖM, M. HÄLlSTEN: Persistent nepotism in peer-review

a)

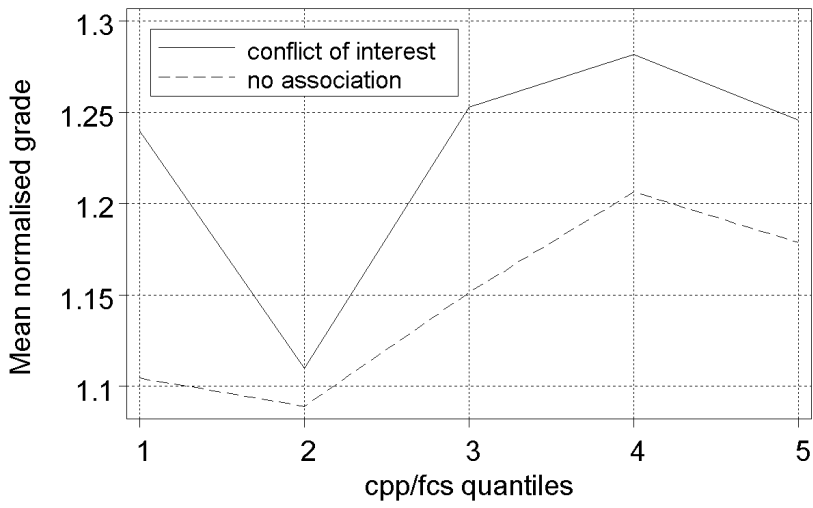

b)

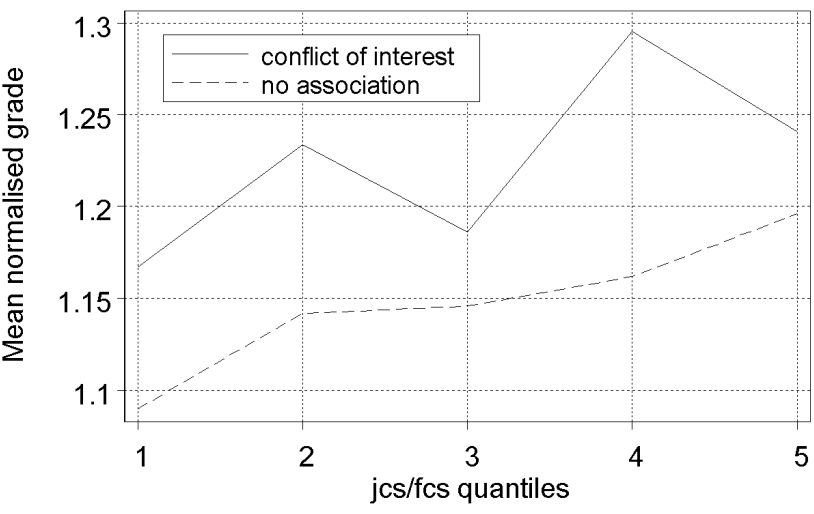

c)

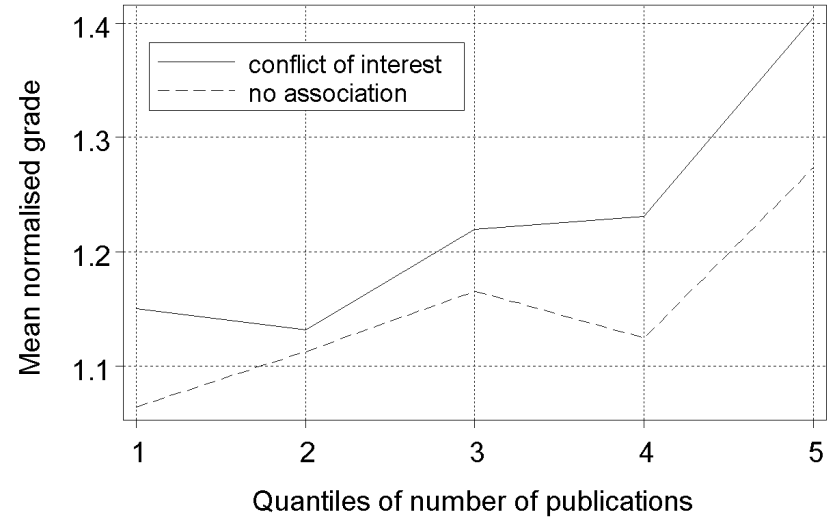

Figure 5. The association between grade and three different productivity measures in quantiles. a) Citations per paper (CPP/JCS); b) Journal Impact (JCS/FCS); c) Number of publications Separate estimates applicants with and without reviewer affiliation 
Second, we examine how grading procedures is related to nepotism in Figures $5 \mathrm{a}-\mathrm{c}$. The pattern is very similar across productivity measures. Principal investigators with reviewer affiliation always receive a higher grade than applicants with no reviewer affiliation. There are no indications of interacting effects.

In the final step, we conduct regression of normalised scores analyses to elucidate the effect of nepotism and female gender. We specify models with control for individual characteristics and several productivity measures. All interaction effects between nepotism, gender and the productivity measures are explored (for descriptive statistics see Table 3 in Appendix). Since we have over-sampled female PIs, we use a weighted linear regression estimator. The results have been thoroughly checked and found robust. We conducted standard regression diagnostics (examination of residuals and influence statistics) and found no evidence that the results are due to the influence of outliers or other mis-specification problems. We also tested alternative specifications of the regression model (i.e. a piecewise linear spline regression model that may handle the nonlinearities of the productivity measures more adequately), but the same conclusions apply.

The explained variance range from 0.35 in the simplest specification to almost 0.5 with a full set of control variables and interactions, which we believe is quite high. In model 1-3 we examine each productivity variable and its interaction with gender separately. The effect of female PI is positive, albeit not significant, but the conflict of interest measure highly significant. It appears that nepotism is persistent in the practices of the research council. Impact score and number of publications influence awarded grades positively, whereas actual citations do not have any influence at all. Surprisingly, none of the productivity measures interact with gender: male and female PIs are judged similarly with reference to productivity. When we control for all productivity measures and interactions in model 4, the positive coefficient for female PIs becomes significant. It appears that female PIs receive a bonus compare to male PIs.

In model 5-7 we examine conflict of interest and its interaction with productivity variables separately. The effect of conflict of interest clearly varies by productivity measures. In model 4, there is no baseline effect of conflict of interest. However, for each publication PIs with research committee association receive a bonus of 0.4 percent. The conflict of interest effect is indifferent to actual citations (model 5). There is a quite strong interaction between impact score and conflict of interest. PIs with low impact have a very large nepotism bonus, whereas high impact PIs receive a lower bonus (it declines with eight percentage points for each normalised impact score). When we control for all productivity measures and interactions in model 8 , only the interaction between conflict of interest and number of publications remain significant. Controlling for all interaction effects in model 9 , the conclusions for model 4 and 8 remain. 
However, the conflict of interest effect is not insignificant, but females receive bonus of around 9 percentage points (point estimate; it is at least 2.4 percentage points according to the confidence interval).

Finally, in model 10, we introduce an interaction term between gender and conflict of interest. The interaction term is significant and negative; the non-significance of the main effect in the previous model was due to a concealed interaction effect. Both the gender and the conflict of interest coefficient are positive and highly significant. We combine the coefficients in Table 1 for all combinations of gender and nepotism. Looking at the point estimates we find that female PIs with association are awarded the same grade as male PIs, whereas, female PIs without association are awarded a higher grade than male PIs. Taking the confidence intervals into consideration, the only significant difference is between males without reviewer affiliation and other applicants.

\section{Discussion}

There is a "productivism bias" in ordinary grant peer review. Our results show that expected impact score and number of publications influence awarded grades positively, whereas actual citations have no influence. The conflict of interest effect increases with number of publications. Males without reviewer affiliation are awarded lower scores than other applicants. The concluding words of WENNERÅs \& WOLD [1997] still apply: "We see no reason why an applicant who manages to produce research of high quality despite not being affiliated with a prestigious research group should not be similarly rewarded." Beyond this, we would conclude that the prestige of peer-review and the Scientific Council for Medicine is under threat if conflicts of interest overshadow the procedures. Still, after ten years, the system seems to be riddled with factors external to science. Nepotism matters.

Research was supported by the Bank of Sweden Tercentenary Foundation, grant no. 97-5085. Bibliometric data from the Swedish Research Council (VR) database. The authors thank Daniel Wadskog (VR) for bibliometric assistance. Data used for this article will soon be published at www.forskningspolitik.se/data.asp. 


\section{References}

Black, M. M., HoldEN, E. W. (1998), The impact of gender on productivity and satisfaction among medical school psychologists, Journal of Clinical Psychology in Medical Settings, 5 (1) : 117-131.

BORNMANN, L., DANIEL, H. D. (2005), Selection of research fellowship recipients by committee peer review. Reliability, fairness and predictive validity of Board of Trustees' decisions, Scientometrics, 63 (2) : 297-320.

BRouns, M. (2000), The gendered nature of assessment procedures on scientific research funding: the dutch case, Higher Education in Europe, 25 : 193-199.

COLE, J. R., ZuCKERMAN, H. (1987), Marriage, Motherhood and Research Performance in Science Scientific American, $256(2): 119$.

GANDER, J. P. (1999), Faculty gender effects on academic research and teaching, Research in Higher Education, 40 (2) : 171-184

GlänZEl, W., DebaCkere, K., Thijs, B., Schubert, A. (2006), A concise review on the role of author selfcitations in information science, bibliometrics and science policy, Scientometrics, 67 (2) : 263-277.

Kulis, S., SicotTe, D., Collins, S. (2002), More than a pipeline problem: Labor supply constraints and gender stratification across academic science disciplines, Research in Higher Education, 43 (6) : 657691.

KyviK, S., TEIGEN, M. (1996), Child care, research collaboration, and gender differences in scientific productivity, Science Technology \& Human Values, 21 (1) : 54-71.

LeVin, S. G., Stephan, P. E. (1998), Gender differences in the rewards to publishing in academe: Science in the 1970s, Sex Roles, 38 (11-12) : 1049-1064.

LONG, J. S. (1992), Measures of sex differences in scientific productivity, Social Forces, 71 (1) : 159-178.

MoEd, H. F. (2005), Citation Analysis in Research Evaluation. Springer Verlag.

PRPIC, K. (2002), Gender and productivity differentials in science, Scientometrics, 55 (1) : 27-58.

Rossiter, M. W. (1993), The Matilda Effect in science, Social Studies of Science, 23 : 325-341.

SAndström, U. \& AL. (1997), “Does Peer Review Matter?” Peers on Peers. Allocations Policy and Review Procedures at TFR. Stockholm, Swedish Research Council for Engineering Sciences.

VAN RAAN, A. F. J. (2006), Statistical properties of bibliometric indicators: Research group indicator distributions and correlations, Journal of the American Society for Information Science and Technology, 57 (3) : 408-430.

WENNERÅs, C., WOLD, A. (1997), Nepotism and sexism in peer-review, Nature, 387 (6631) : 341-343.

WESSELY, S. ( 1998), Peer review of grant applications: what do we know? Lancet, 352 (9124) : 301-305.

WOLD, A., ChraPKOwSKA, C. (2004), Förbjuden frukt på kunskapens träd. Atlantis. [in Swedish]

XiE, Y., Shauman, K. A. (1998), Sex differences in research productivity: New evidence about an old puzzle, American Sociological Review, 63 (6) : 847-870. 


\section{Appendix}

Table 3. Descriptive statistics

\begin{tabular}{|c|c|c|c|c|c|c|c|}
\hline Variable & observations & mean & stddev & $\min$ & $\max$ & percent & number \\
\hline granted application & 280 & & & 0 & 1 & 11.1 & 31 \\
\hline normalised grade score & 280 & 1.163 & 0.178 & 0.669 & 1.676 & & \\
\hline conflict of interest & 280 & & & 0 & 1 & 21.4 & 59 \\
\hline principal investigator is female & 280 & & & 0 & 1 & 42.1 & 118 \\
\hline quality (cpp/fcs) & 280 & 1.203 & 0.878 & 0 & 10.899 & & \\
\hline expected impact (jcs/fcs) & 280 & 1.128 & 0.364 & 0.324 & 2.356 & & \\
\hline number of publications & 280 & 16.686 & 15.231 & 1 & 108 & & \\
\hline assistant professor & 280 & & & 0 & 1 & 35 & 97 \\
\hline professor & 280 & & & 0 & 1 & 21.1 & 59 \\
\hline experience & 280 & 11.821 & 8.145 & 0 & 41 & & \\
\hline medical faculty & 280 & & & 0 & 1 & 68.2 & 191 \\
\hline university: GU & 280 & & & 0 & 1 & 11.8 & 32 \\
\hline university: LIU & 280 & & & 0 & 1 & 9.3 & 25 \\
\hline university: LU & 280 & & & 0 & 1 & 9.6 & 27 \\
\hline university: UMU & 280 & & & 0 & 1 & 8.9 & 24 \\
\hline university: UU & 280 & & & 0 & 1 & 7.5 & 21 \\
\hline university: other & 280 & & & 0 & 1 & 13.2 & 37 \\
\hline committee: health and care & 280 & & & 0 & 1 & 17.9 & 49 \\
\hline committee: clinical science & 280 & & & 0 & 1 & 17.9 & 49 \\
\hline committee: medical chemistry & 280 & & & 0 & 1 & 7.9 & 21 \\
\hline committee: microbiology & 280 & & & 0 & 1 & 17.9 & 49 \\
\hline committee: psychiatry & 280 & & & 0 & 1 & 8.9 & 24 \\
\hline committee: pharmacology & 280 & & & 0 & 1 & 12.1 & 34 \\
\hline
\end{tabular}

